\title{
MECHANISMS OF BONE METASTASIS OF MALIGNANCIES OF THE GENITO-MAMMARY AREA
}

doi: 10.2478/rojost-2018-0053

\author{
R. Bohîlțea ${ }^{1,2}$, N. Turcan'2, T.A. Georgescu3, M.M. Cîrstoiu'1,2 \\ "Carol Davila" University of Medicine and Pharmacy, Bucharest, Romania \\ ${ }^{2}$ Department of Obstetrics and Gynecology, University Emergency Hospital, Bucharest, Romania \\ 3Department of Pathological Anatomy, "Carol Davila" University of Medicine and Pharmacy, \\ Bucharest, Romania
}

\begin{abstract}
Bone metastasis is a frequent complication of advanced genito-mammary cancer patients. Skeletal involvement is particularly common in breast cancer. Bone metastases induce a wide range of symptoms, lowering the quality of life and shortening survival. The normal bone remodeling process is deeply affected in all types of metastases: osteolytic, osteoblastic, and mixed. The main mechanisms involved in bone metastatic dissemination are the expression of adhesion tumor molecules and corresponding receptors within bone marrow and bone matrix cells; local growth factors, molecular mechanisms of remodeling the hematopoietic stem cell activity, and alteration of the expression of the post-transcriptional regulatory microRNAs of the gene expression are the new theories developed from recent studies.
\end{abstract}

\begin{abstract}
Abnormalities in the number of copies of the $16 q 23$ gene explain the increased risk of bone metastasis of breast cancer compared to its dissemination to the other organs; the mutual interaction between tumor cells and the bone microenvironment constitutes the element that stimulates both bone destruction and tumor development. Endothelin -1 , bone morphogenic proteins, platelet-derived growth factor, Wnt proteins stimulate proliferation and osteoblastic activity. Genomic and proteomic studies underlie the development of new therapeutic agents for the treatment and prevention of bone metastases.

Keywords: bone metastases, genomics, proteomics
\end{abstract}

